\title{
THE
}

$12-18-1978$

\section{Observation of Newtonian Capillary Shear Flow in an Oriented Cholesteric Liquid Crystal}

\author{
S. Bhattacharya \\ C. E. Hong \\ S. V. Letcher \\ University of Rhode Island, sletcher@uri.edu
}

Follow this and additional works at: https://digitalcommons.uri.edu/phys_facpubs

Terms of Use

All rights reserved under copyright.

\section{Citation/Publisher Attribution}

Bhattacharya, S., Hong, C. E., \& Letcher, S. V. (1978). Observation of Newtonian Capillary Shear Flow in an Oriented Cholesteric Liquid Crystal. Physical Review Letters, 41(25), 1736-1739. doi: 10.1103/

PhysRevLett.41.1736

Available at: http://dx.doi.org/10.1103/PhysRevLett.41.1736

This Article is brought to you for free and open access by the Physics at DigitalCommons@URI. It has been accepted for inclusion in Physics Faculty Publications by an authorized administrator of DigitalCommons@URI. For more information, please contact digitalcommons-group@uri.edu. 
tures in He II with a factor of about 3.5 at a power level of $1.0 \mathrm{~W} / \mathrm{mm}^{2}$.

Following the same reasoning, one might be tempted to argue that the change in slope in the converted $\mathrm{T}$ mode at $0.8 \mathrm{~W} / \mathrm{mm}^{2}$ (Fig. 2) could be related to the minimum energy $(\sim 8 \mathrm{~K})$ for direct coupling the phonon flux to roton excitations in He II. Because of the black-body radiation involved, the effect of the 8-K energy levels is expected to show up at frequencies $\omega=2.8 k T / \hbar$, where $T$ is the effective pulse temperature. Taking into account the above mentioned scaling down of the temperatures, the break in slope appears at approximately the right energy for direct roton excitation. However, the rather sharp break in slope is not to be expected on the basis of a broad Planck distribution.

The results from our phonon-transmission experiments show clearly a changed L/T ratio found after propagation through the interface, as compared with this ratio in the solid crystal. The observed pressure dependence indicates that during conversion the frequency distribution (effective pulse temperature) of the L-mode phonon pulse is more strongly affected than that of the T-mode phonon pulse. Obviously, these first experiments should be refined with use of tunnel junctions as quasimonochromatic phonon generators and detectors and by better surface characterization.
We are most grateful to Dr. J. Wolter for interesting discussions on this subject. Part of this work has been supported by the Stichting voor Fundamenteel Onderzoek der Materie (FOM) with financial support of the Nederlandse Organisatie voor Zuiver Wetenschappelijk Onderzoek (ZWO).

${ }^{1}$ G. L. Pollack, Rev. Mod. Phys. 41, 48 (1969).

${ }^{2}$ L. J. Challis, J. Phys. C 7, 481 (1974).

${ }^{3}$ J. Weber, W. Sandmann, W. Diets che, and H. Kinder, Phys. Rev. Lett. 40, 1469 (1978).

${ }^{4}$ R. J. von Gutfeld and A. Nethercot, Jr., Phys. Rev. Lett. 12, 641 (1964).

${ }^{5}$ H. J. Maris, B. Taylor, and C. Elbaum, Phys. Rev. B 3, 1462 (1971).

${ }^{6}$ C. J. Guo and H. J. Maris, Phys. Rev. Lett. $\underline{29}, 855$ (1972).

${ }^{7}$ R. A. Sherlock, A. F. G. Wyatt, N. G. Mills, and

N. A. Lockerbie, Phys。Rev. Lett. 29, 1299 (1972).

${ }^{8}$ T. J. B. Swanenburg and J. Wolter, Phys. Rev. Lett. 31, 693 (1973).

${ }^{9}$ K. Andres, R. C. Dynes, and V. Narayanamurti, Phys. Rev. Lett. 31, 687 (1973).

${ }^{10} \mathrm{O}$. Weis, Z. Angew. Phys. 26, 325 (1969).

${ }^{11}$ J. Maynard, Phys. Rev. B 14,3868 (1976).

${ }^{12}$ F. Rösch and O. Weis, Z. Phys. B29, 71 (1978).

${ }^{13}$ R. C. Dynes and V. Narayanamurti, Phys. Rev. B 12,1720 (1975).

${ }^{14}$ A. F. G. Wyatt, N. A. Lockerbie, and R. A. Sherlock, Phys. Rev. Lett. 33, 1425 (1974).

${ }^{15}$ R. A. Sherlock, A. F. G. Wyatt, and N. A. Lockerbie, J. Phys. C 10, 2567 (1977).

\title{
Observation of Newtonian Capillary Shear Flow in an Oriented Cholesteric Liquid Crystal
}

\author{
S. Bhattacharya, C. E. Hong, and S. V. Letcher \\ Physics Department, University of Rhode Island, Kingston, Rhode Island 02881, and Francis Bitter National \\ Magnet Laboratory, Massachusetts Institute of Technology, Cambridge, Massachusetts 02139
}

(Received 26 October 1978)

\begin{abstract}
Newtonian capillary shear flow was observed for the first time in a cholesteric liquid crystal with the helix axis perpendicular to the flow. The effective viscosity in the cholesteric phase had a magnitude comparable to the viscosity of the isotropic liquid extrapolated to the cholesteric temperature. Oscillatory pitch dependence of the viscosity was seen when the pitch length was comparable to the capillary width.
\end{abstract}

Early experimental studies of the capillary shear flow properties of unoriented cholesteric liquid crystals found strongly non-Newtonian behavior with the apparent viscosity at low shear rates several orders of magnitude larger than the isotropic phase values. ${ }^{1}$ This was explained by Helfrich's model of a permeation mechanism ${ }^{2}$ that inhibits the flow when the helix axis is paral- lel to the direction of flow. Lubensky ${ }^{3}$ derived the same sort of mechanism for flow parallel to the pitch axis from a hydrodynamic theory。 Subsequently, it was shown by Leslie ${ }^{4}$ that capillary shear flow should be Newtonian for flow perpendicular to the helix axis and the effective viscosity should be given by a combination of the Leslie nematic vescosity coefficients. A somewhat more 
detailed theoretical study of this configuration was done recently by Kini. ${ }^{5}$

We present here the first evidence of a Newtonian capillary shear flow in a cholesteric liquid crystal oriented with the helix axis perpendicular to the flow. The material chosen was a mixture of cholesteryl chloride and cholesteryl myristate, $1.65: 1$ by weight. This mixture has a negative magnetic anisotropy ${ }^{6}$ and thus aligns with the helix axis along the field direction. Furthermore, the components have opposite helicity, which results in the pitch of the mixture becoming infinite at $\sim 52^{\circ} \mathrm{C}$ (hereafter referred to as $T_{N}$ )。

The capillary shear flow was measured by a flat capillary of rectangular cross section (width $=180 \mu \mathrm{m}$ ). The experimental geometry is shown in Fig. 1. The inner surfaces were rubbed along the vertical direction to obtain homogeneous alignment at the surface. A magnetic field of 64 kOe was applied perpendicular to the surfaces in the horizontal plane. The flow was in the vertical direction so that the helix axis remained normal to the flow direction. The temperature of the capillary was controlled by a circulating water bath to within $\pm 0.05 \mathrm{~K}$ 。 The method of measuring the relative changes in viscosity by a rectangular capillary is described elsewhere. ${ }^{7}$ The absolute values were determined in the isotropic phase with a calibrated Ubbelohde viscometer.

The flow was found to be Newtonian in the cholesteric phase. The viscosity was independent of pressure (within our resolution of $1 \%$ ) in the pressure range $20-400 \mathrm{~mm}$ of water head, which corresponds to shear stress at the capillary walls of

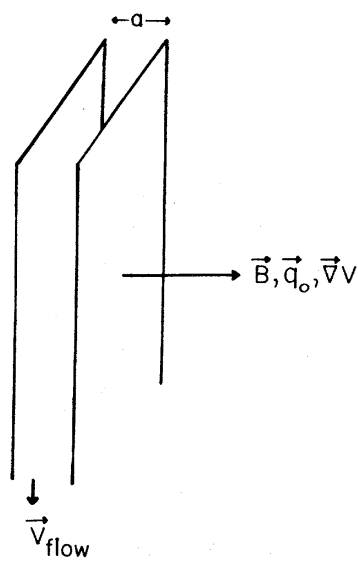

FIG. 1. Geometry of flow. The magnetic field $\vec{B}$ is parallel to the pitch axis $q_{0}$ and perpendicular to $\vec{v}_{\mathrm{flo}}$. The molecules at each surface are vertical.
$3.5-70 \mathrm{dyn} / \mathrm{cm}^{2}$ and shear rates on the order of $10 \mathrm{sec}^{-1}$. The magnetic field was strong enough to preserve orientation during flow and possible non-Newtonian effects arising from flow alignment were avoided (except near $T_{N}$-see below).

The temperature dependence of the measured viscosity is shown in Fig. 2. We make the following observations:

(1) Near the isotropic-cholesteric transition a decrease in viscosity occurs on entering the cholesteric phase. This is exactly the behavior observed at the isotropic-nematic transition ${ }^{7,8}$ for the two nematic geometries (nematic director parallel to the flow and director normal to both the flow velocity and the velocity gradient) that correspond to this cholesteric orientation. It is in contrast with the kind of peak observed with rotational viscometers and unoriented samples. ${ }^{9}$

(2) On both sides of the isotropic-cholesteric transition the flow is Newtonian. This contrasts with previous observations ${ }^{9}$ of strong non-Newtonian effects near the transition for unoriented samples.

(3) The temperature dependence of the viscosity in the cholesteric phase exhibits Arrhenius behavior, with the activation energy below $T_{N}$ being significantly larger than that above $T_{N}$ (29 and $11.5 \mathrm{kcal} / \mathrm{mole}$, respectively).

(4) The magnitude of the viscosity in the cholesteric phase is of the same order as the extrapolated value from the isotropic phase. The large values of viscosity we observe in this oriented sample are, then, due more to the molecular configuration and interactions than to the cooperative

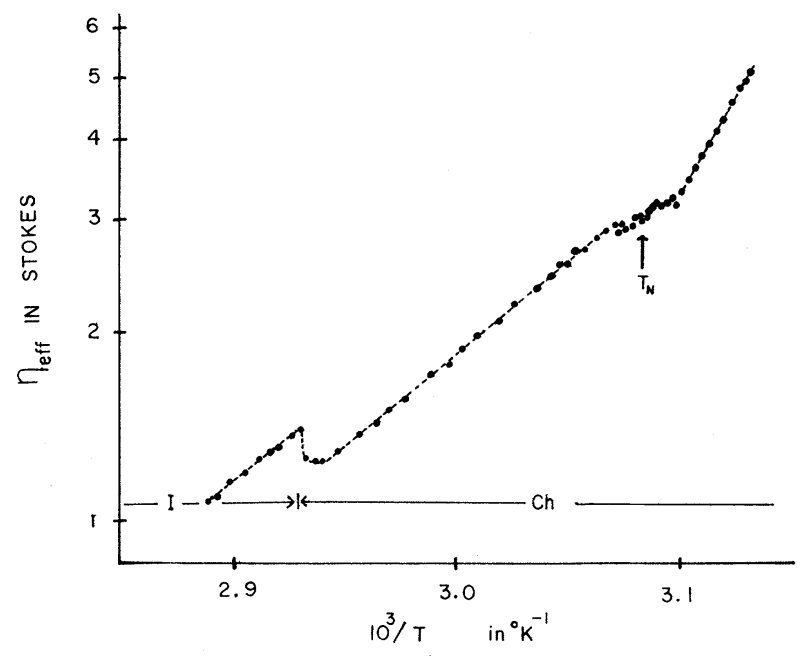

FIG. 2. Temperature dependence of the measured viscosity in the isotropic and cholesteric phases. 
cholesteric structure. The abnormally large capillary viscosities reported in unoriented cholesterics can be explained by Helfrich's permeation effect, as stated earlier.

(5) Near $T_{N}$ the viscosity departs from the Arrhenius behavior and exhibits an oscillary temperature dependence. A careful study of the behavior near $T_{N}$ is shown in Fig. 3. A markedly oscillatory temperature dependence is observed.

In order to explain this seemingly anomalous behavior near $T_{N}$, we use the theoretical calculations of Leslie ${ }^{4}$ and $\mathrm{Kini}^{5}$. Ignoring the thermomechanical coupling, the effective viscosity in our geometry is given by

$$
\eta_{\text {eff }}=\frac{2 \alpha_{4}\left(\alpha_{4}+\alpha_{3}+\alpha_{6}\right)}{\left(2 \alpha_{4}+\alpha_{3}+\alpha_{6}\right)-\left(\alpha_{3}+\alpha_{6}\right) \sin q_{0} a / q_{0} a},
$$

where $\alpha_{i}$ are the Leslie coefficients, $q_{0}=2 \pi / P$, where $\boldsymbol{P}$ is the pitch, and $a$ is the width of the capillary. Far away from $T_{N}$, the pitch is very small and the last term in the denominator can be neglected. Then the viscosity becomes

$$
\eta_{\text {eff }}=\frac{2 \alpha_{4}\left(\alpha_{4}+\alpha_{3}+\alpha_{6}\right)}{2 \alpha_{4}+\alpha_{3}+\alpha_{6}}=2\left[\eta_{a}^{-1}+\eta_{c}^{-1}\right]^{-1},
$$

which is the appropriate average of the two Miesowicz ${ }^{10}$ nematic viscosities: $\eta_{a}=\alpha_{4}$ and $\eta_{c}=\alpha_{4}+\alpha_{3}$ $+\alpha_{6}$, one for flow parallel to the local director and the other for director perpendicular to both the flow velocity and the velocity gradient. In this region the viscosity is independent of the pitch.

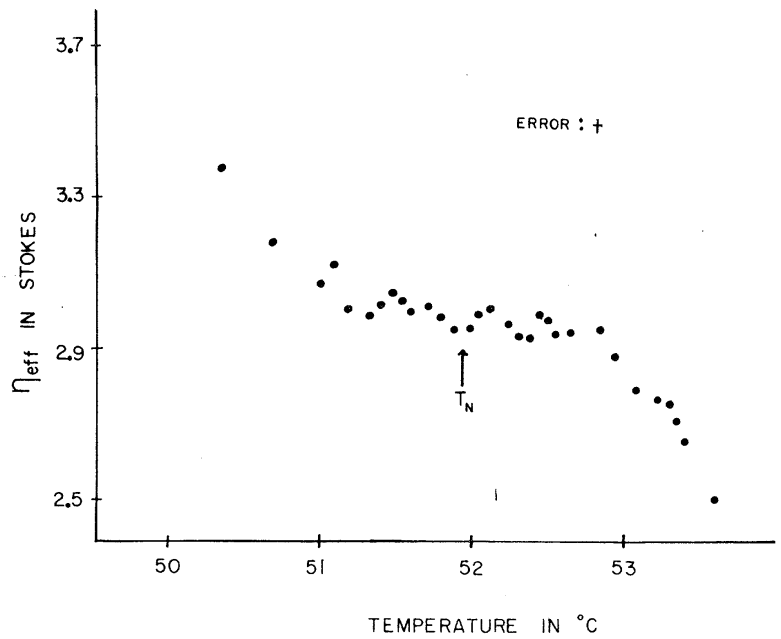

FIG. 3. Temperature dependence of the effective viscosity near $T_{N}$, the nematic point. The value of $\boldsymbol{T}_{N}$ was measured using a polarizing microscope, but the exact value shown was chosen as the minimum of the broadest oscillation.
Near $T_{N}$, however, the last term in the denominator in Eq. (1) is no longer small and the oscillatory behavior of the $\sin \left(q_{0} a\right)$ term contributes to the oscillation of the viscosity with pitch, which in our case is realized by temperature change. The first maximum of the viscosity occurs at $q_{0} a \simeq 4.48$ [solution of the transcendental equation $\tan \left(q_{0} a\right)=q_{0} a$ ]。

From previous experiments, ${ }^{11}$ the temperature dependence of the pitch for this mixture was found to be given by $P=P_{0} /\left|T-T_{N}\right|$, where $P_{0}$ $=42 \mu \mathrm{m} \mathrm{K}$. From this we can calculate the temperature equivalent of the viscosity oscillations. The first maximum is found to occur at $0.17 \mathrm{~K}$, which compares well with our measured value of $\sim 0.2 \mathrm{~K}$. Since it is not known why the activation energies above and below $T_{N}$ are so different nor how the crossover between the two regimes takes place, it is not possible to subtract the background and fit the oscillatory behavior of the viscosity to $\mathrm{Eq}$. (1). However, we believe that our good semiquantitative agreement indicates that the theoretical treatments are basically correct.

Near $T_{N}$, the viscosity is lightly non-Newtonian; the viscosity changes by $2 \%$ in going through one order of magnitude change in the pressure differ ence. This we attribute to flow alignment. Recall that the molecular axes are normal to the magnetic field and, therefore, flow alignment in the plane normal to the field is not inhibited by the field. It is, however, thoroughly inhibited by the cholesteric structure, except near $T_{N}$, where the pitch length becomes very large. Near $T_{N}$ the flow can change the molecular alignment and result in non-Newtonian behavior.

We repeated these measurements in the same surface-treated, flat capillary without a magnetic field. In the cholesteric phase the flow becomes non-Newtonian and the effective viscosity has larger values at lower shear rates. This is qualitatively consistent with previous capillary flow experiments in a cylindrical geometry. ${ }^{12}$ The effect is more pronounced as the temperature is lowered. However, the boundary alignment is found to be strong and the flow alignment does not distort the structure very much. This is further evidenced by applying a magnetic field in the vertical direction along the flow. At $65^{\circ} \mathrm{C}$, a field of $135 \mathrm{kOe}$ produces a $50 \%$ increase of the effective viscosity, but no saturation effect is seen, indicating that the field is not sufficient to produce complete disorientation with respect to the boundary alignment. The non-Newtonian behavior is, of course, much stronger. 
Capillary shear flow was also measured in an untreated viscometer of circular cross section (diam $=2 \mathrm{~mm}$ ) in a vertical field; $i_{0} e_{\text {, }}$, with the flow along the helix axis. In this geometry the fluid becomes nearly immobile at lower shear rates, yielding apparent viscosities several orders of magnitude higher than in the isotropic phase. In the absence of the field the flow is still non-Newtonian but the effective viscosity is smaller, indicating that the field helps produce substantially more orientation of the helix axis along the flow. This supports the Helfrich permeation model. Attempts to force flow with high pressure yield a significantly smaller effective viscosity, which we interpret to be a consequence of defect flow. The system does not easily recover from the defect structure; large hysteresis effects are present. The structure is found to cure only after heating to the isotropic phase.

In conclusion, we have observed, for what we believe is the first time, the following confirmations of theoretical predictions: (1) Newtonian capillary shear flow in a cholesteric for flow perpendicular to the helix axis; (2) Newtonian flow near the isotropic-cholesteric phase transition;
(3) an oscillatory temperature dependence of the viscosity near $T_{N}$ 。

This work was supported by the National Science Foundation under Grant No。 DMR77-07813。

\footnotetext{
${ }^{1}$ R. S. Porter, E. M. Barrall, and J. F. Johnson, J. Chem. Phys. 45, 1452 (1966).

${ }^{2}$ W. Helfrich, Phys. Rev. Lett. 23,372 (1969).

${ }^{3}$ T. C. Lubensky, Phys. Rev.A $\underline{6}, 452$ (1972).

${ }^{4}$ F. M. Leslie, Mol. Cryst. Liquid Cryst. $\underline{7}, 407$ (1969).

${ }^{5}$ U. D. Kini, to be published.

${ }^{6}$ E. Sackmann, S. Meiboom, L. C. Snyder, A. E. Meixner, and R. E. Dietz, J. Am. Chem. Soc. 90, 3567 (1968).

${ }^{7}$ M. G. Kim, S. Park, Sr., M. Cooper, and S. V. Letcher, Mol. Cryst. Liquid Cryst. 36, 143 (1976). ${ }^{8} \mathrm{Ch}$. Găhwiller, Mol. Cryst. Liquid Cryst. 20,301 (1973).

${ }^{9}$ T. Yamadà and E. Fukuda, Jpn. J. Appl. Phys. 12, 68 (1973).

${ }^{10}$ M. Miesowicz, Nature (London) 158, 27 (1946).

${ }^{11} \mathrm{H}$. Baessler and M. M. Labes, J. Chem. Phys. $\underline{52}$, 631 (1970).

${ }^{12}$ S. Candau, P. Martinoty, and F. Debeauvais, C. R. Acad. Sci., Ser.A 277, 769 (1973).
}

\title{
Optically Detected Coherent Transients in Nuclear Hyperfine Levels
}

\author{
R. M. Shelby, C. S. Yannoni, and R. M. Macfarlane \\ IBM Research Laboratory, San Jose, California 95193
}

(Received 17 October 1978)

\begin{abstract}
Using optical detection, we have observed rf nutation, free-induction decay, and echoes, on the 16.70- $\mathrm{MHz}$ nuclear hyperfine transition of dilute $\mathrm{Pr}^{3+}$ in $\mathrm{LaF}_{3}$ at $2 \mathrm{~K}$. From a comparison of free-induction decay and echo decay we find that the previously observed linewidth of $200 \mathrm{kHz}$ is inhomogeneous and that the homogeneous width is $19 \mathrm{kHz}$. On the basis of these measurements it is suggested that optical dephasing times for the ${ }^{1} \mathrm{D}_{2}-{ }^{3} \mathrm{H}_{4}$ and ${ }^{3} P_{0}-{ }^{3} H_{4}$ transitions are as long as $\sim 20 \mu \mathrm{sec}$.
\end{abstract}

Coherent-transient techniques in pulsed NMR have proven themselves in recent years to be a very effective means of studying magnetic interactions in solids. ${ }^{1}$ In particular they have elucidated the way in which these interactions contribute to homogeneous and inhomogeneous broadening, ${ }^{1,2}$ thus allowing high-resolution measurements to be made in inhomogeneous environments.

It is known that optical detection can substantially improve the sensitivity of magnetic-resonance experiments, providing a simple method of studying dilute-spin systems. ${ }^{3}$ This idea has found widespread application in $\mathrm{ESR}^{3}$ and electron-nuclear double resonance ${ }^{4}$ and has recently been demonstrated in solid-state NMR. ${ }^{5}$ In this paper we report the extension of optical-detection techniques to the study of rf coherent transients in nuclear hyperfine levels. Because of its greatly enhanced sensitivity, it should significantly expand the application of these techniques to dilute-spin systems where nuclear polarization can be induced by optical pumping.

We have chosen to illustrate the se techniques with a system of great current interest, viz. $\mathrm{LaF}_{3}: \mathrm{Pr}^{3+}$. Optical-dephasing (i.e., $T_{2}$ ) measurements have been carried out on ${ }^{1} \mathrm{D}_{2} \rightarrow{ }^{3} \mathrm{H}_{4}$ (Refs. 6-8) and ${ }^{3} P_{0} \rightarrow{ }^{3} H_{4}$ (Refs. 8-10) transitions using a variety of laser techniques. Although laser 